\title{
Design of Quick-Dry Surface Using Structured Surface*
}

\author{
Daisuke ARUGA $^{* *}$, Arata KANEKO ${ }^{* *}$ and Nobuyuki MORONUKI ${ }^{* *}$ \\ "Graduate school of System Design, Tokyo Metropolitan University, \\ 6-6, Asahigaoka, Hino, Tokyo, 191-0065, Japan \\ E-mail: kaneko-arata@tmu.ac.jp
}

\begin{abstract}
This paper discusses the design of surface microstructures for quick drying. The structures produced in this study were arrays of micropillars with different sizes and pitches fabricated by photolithography. It was found that the sliding angle decreased with an increase in the pitch of the pillars. Hence, droplets could be removed easily. However, the evaporation time increased significantly. To achieve a balance between the evaporation time and the sliding angle, a reticular pattern consisting of pillars and flat areas was designed and tested. The evaporaiotn time became shorter than the fully arrayed surface on this pattern, while the sliding angle could not be made small. The evaporation time became shorter than the fully arrayed surface on this pattern, while the sliding angle could not be made small.
\end{abstract}

Key words: Structured Surface, Wettability, Quick Dry, Sliding Angle, Evaporation

\section{Introduction}

A textured surface or a structured surface can be used for various purposes, e.g., to control wettability and friction and to produce an antireflective surface. Wettability can be controlled to produce a superhydrophobic surface, reduce flow resistance, etc. ${ }^{(1)-(5)}$. Quick-dry performance, which is achieved by controlling wettability, of materials used to produce windshields of vehicles, tiles for bathroom floors, etc., must be high. However, the design method to obtain a quick-dry surface has not yet been developed since various issues should be taken into account such as wetting, spreading, evaporation, etc..

Two parameters, namely, the sliding angle and the evaporation time, can be used evaluate the quick-dry performance. The sliding angle is the angle at which a droplet begins to slide down an inclined surface. The smaller the sliding angle, the easier it is to remove droplets from the surface. The evaporation time is the time taken by droplets to evaporate from the surface. It can be estimated by measuring the decrease in the mass of droplets with time. A superhydrophobic surface has a small sliding angle ${ }^{(6)}$; however, in this case, the evaporation time is long since the surface area of droplets is small ${ }^{(7)}$.

In this study, we prepared a structured surface that consisted of an array of pillars of different sizes and pitches and we produced an appropriate design of a microstructured surface for quick drying.

\section{Basics of wettability}

Figure 1 (a) shows the cross section of a droplet on a surface. The radius of the droplet is smaller than the capillary length described below. The contact angle $\theta$ takes a specific value that is suitable for balancing three interfacial tensions. It can be given by Young's equation as follows ${ }^{(8)}$ : 


$$
\cos \theta=\frac{\gamma_{S V}-\gamma_{S L}}{\gamma_{L V}}
$$

where $\gamma_{S V}, \gamma_{S L}$, and $\gamma_{L V}$ are the interfacial tensions per unit area of the solid-vapor, solid-liquid, and liquid-vapor interfaces, respectively. These tensions are determined by a combination of liquid, vapor, and solid materials used.

When an external force (e.g., gravity force) is applied, $\theta$ changes to balance the external force, as shown in Fig. 1 (b). Thus, the contact angle on one side of the droplet differs from that on the other side; in other words, two contact angles, namely, an advancing angle $\theta_{A}$ and a receding angle $\theta_{R}$, are formed. The relationship between the sliding angle $\alpha$ and the contact angles is given as follows:

$$
\sin \alpha=\frac{\gamma_{L V}}{\rho g V}\left(\cos \theta_{R}-\cos \theta_{A}\right)
$$

where $\rho, g$, and $V$ are the density of liquid, gravitational acceleration, and volume of the droplet, respectively. The difference between $\theta_{A}$ and $\theta_{R}$ becomes constant the droplet slides down the inclined surface. This difference should be minimized in order to obtain a surface on which droplets can slide down easily.

When the radius of the droplet is larger than the capillary length as shown Fig. 1 (c), the top of the droplet becomes flat due to the effect of gravity. The length of the flat area is determined from the capillary length $\kappa^{-1}$, which is given as follows:

$$
\kappa^{-1}=\sqrt{\frac{\gamma_{L V}}{\rho g}}
$$

If the radius of the droplet is larger than $\kappa^{-1}$, gravity force flattens the droplet.

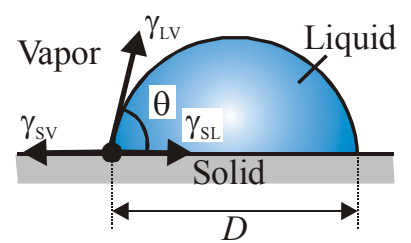

(a) Contact angle $\left(D / 2<\kappa^{-1}\right)$

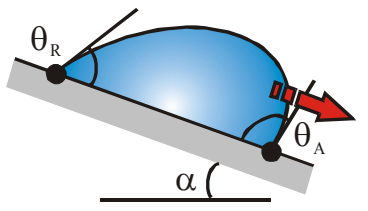

(b) Sliding angle $\left(D / 2<\kappa^{-1}\right)$

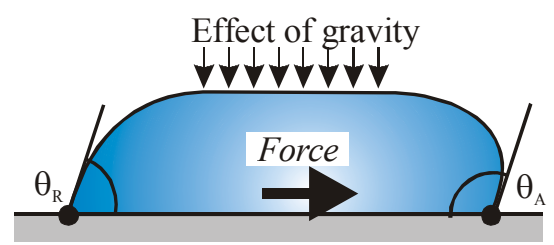

(c) Motion of droplet $\left(D / 2>\kappa^{-1}\right)$

Fig. 1 Basic theory of wetting

From the standpoint of quick-dry performance, the profile of a droplet is important. To decrease the evaporation time of a droplet, it is necessary to increase the surface area exposed to air ${ }^{(9)}$. However, the surface area decreases during evaporation. It is possible to increase the surface area by applying the following method.

Figure 2 shows movement and distortion of the contact line when a substrate with a pillar is moved upward from water ${ }^{(10)}$. It is found that contact line is strongly distorted at the pillar. The lower edge of the pillar serves as a pin. After the pillar is lifted to a certain 
height, it is observed that the contact line drops to the original level. Since the lower edge of the pillar serves as a pin, this phenomenon is called pinning and is often observed on a hydrophilic surface. The force $F$ required to remove the contact line from the pillar per unit length can be given as

$$
F \equiv \gamma_{L V}\left(\cos \theta_{P}-\cos \theta\right)
$$

where $\theta_{P}$ is the contact angle in the case of pinning. The contact line does not move until the contact angle is below a certain value. Since it is possible to increase the surface area with the elongation of the droplet, the surface area in the case of pinning is larger than that in the case of no pinning.

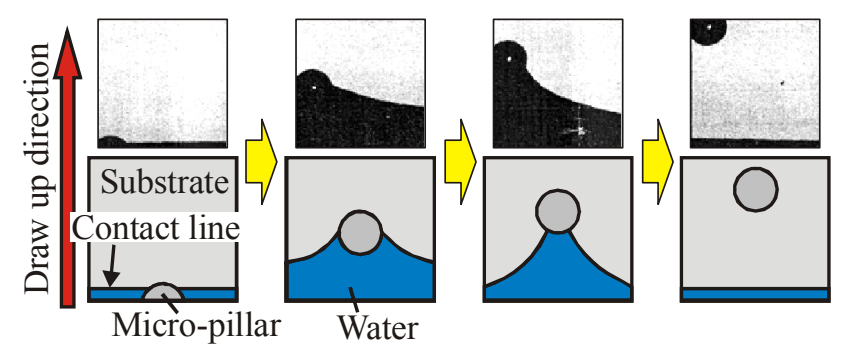

Fig. 2 Pinning at the contact line by pillar ${ }^{(10)}$

Figure 3 shows droplets with and without pinning. Since an additional force is necessary to move the droplets in the case of pinning, $\theta_{R}$ is less than that in the case of no pinning and the sliding angle increases $\left(\alpha<\alpha_{P}\right)$. Thus, pinning should be avoided in order to remove droplets under sliding motion that is necessary to make quick-dry surface. An appropriate design for quick-dry surface should compromise these inconsistent conditions of pinning. Thus, systematic experiments are necessary.

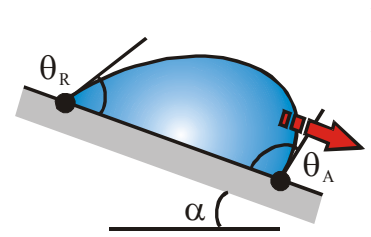

(a) Non-pinning of droplet

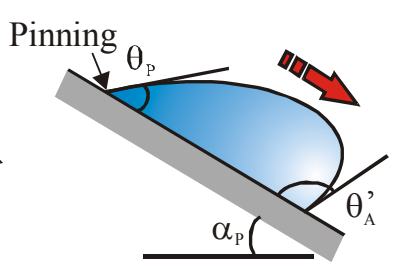

(b) Pinning of droplet

Fig. 3 Sliding angle of droplet with and without pinning

\section{Experimental procedure}

\subsection{Preparation of substrates}

Structures were prepared by photolithography using a negative photoresist SU-8 (MicroChem Corp.). SU-8 is used extensively because it allows us to produce microstructures with a high aspect ratio $(>7)$ and excellent mechanical properties ${ }^{(11)}$. The structures were arrays of pillars of different sizes and pitches. SU-8 has a hydrophilic characteristic: the contact angle for pure water is approximately $70^{\circ}$ on a flat surface. This hydrophilic characteristic enhances pinning ${ }^{(10)}$.

Figure 4 shows the fabrication process of the pillars. SU-8 was spin-coated on a silicon wafer and its whole area was exposed. Then, SU-8 was recoated on the first layer and exposed through a mask with a pattern. Finally, the substrate was developed. Using this process, the entire structure was made of SU-8. Figure 5 shows an SEM image of the 
fabricated pillars together with specific parameters, namely, the diameter $D$, pitch $P$, and height $H$.

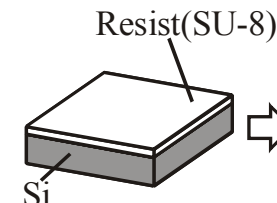

(a) Resist coating (first layer)

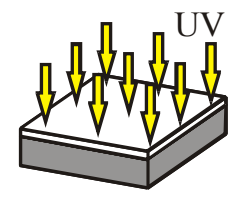

(b) Exposure

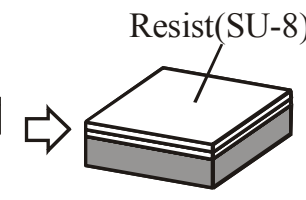

(c) Resist coating (second layer)

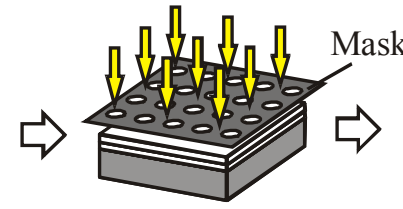

(d) Exposure

(e) Development

Fig. 4 Fabrication process of pillars

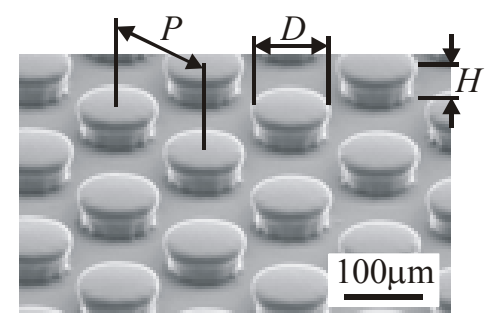

Fig. 5 SEM image of pillars

\subsection{Definition of sliding angles and their measurement}

Figure 6 shows the sliding behavior of a droplet. When the substrate is inclined, the advancing line starts to move, and further inclination triggers the motion of the receding line. The angles of inclination thus formed are defined as advancing sliding angle $\alpha_{A}$ and receding sliding angle $\alpha_{R}$, respectively. The smaller the value of $\alpha_{A}$, the easier it is for the droplet to spread. On the other hand, $\alpha_{R}$ is related to the sliding of the droplet. Thus, the smaller the value of $\alpha_{R}$, the easier it is for the droplet to slide. A large difference between these angles distorts the droplet profile and increases the surface area; therefore, it will shorten the evaporation time.

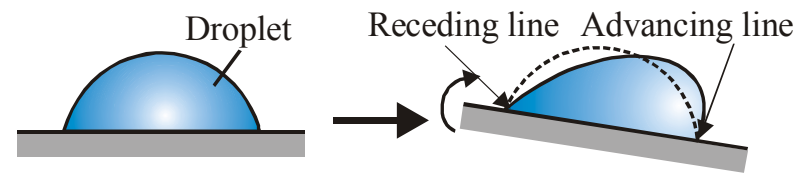

(a) Droplet on solid surface

(b) Deformation

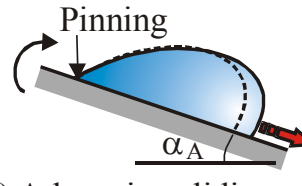

(c) Advancing sliding angle (Elongation of droplet)

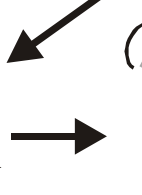

(d) Receding sliding angle (Sliding of droplet)

Fig. 6 Definition of sliding angles

The values of $\alpha_{A}$ and $\alpha_{R}$ were measured by using the setup shown in Fig. 7. A droplet with a definite volume was dropped on a sample with a microstructure using the motorized 
syringe. The contact angle was measured by a combination of a CCD camera and processing software. The sample stage was inclined by tilting the entire system. Thus, the sliding angles were measured by determining the contact angle and inclining the system. The thermal effect of light on the droplet could be neglected.

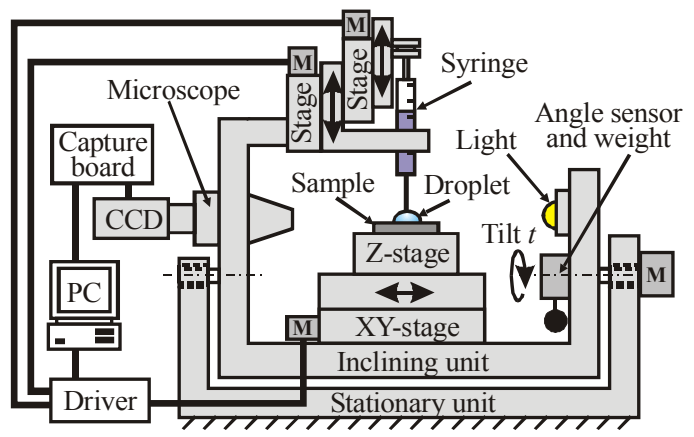

Fig. 7 Setup for sliding angle measurement

\subsection{Setup for measurement of evaporation time}

The evaporation time is the time required for the weight of the droplet to become zero through evaporation. Figure 8 shows the setup for the measurement of the evaporation time. The apparatus was installed in a chamber to eliminate any disturbance (contamination or wind). The mass of the droplet was measured using an electric balance. A CCD camera was set above the droplet to observe its profile during evaporation.

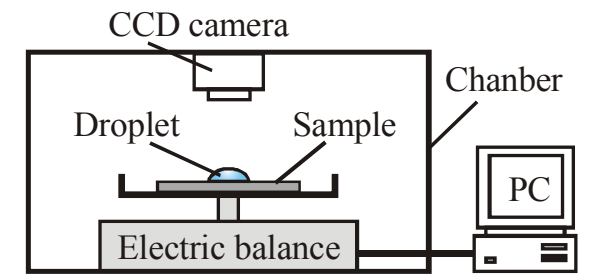

Fig. 8 Setup for measurement of evaporation time

\subsection{Experimental conditions}

Table 1 shows the experimental conditions. All experiments were conducted in a clean room by controlling the temperature and humidity. The temperature was maintained at $20{ }^{\circ} \mathrm{C}$. The radius of the droplets was larger than the capillary length of pure water $\left(\kappa^{-1}=\right.$ $2.7 \mathrm{~mm}$ ); therefore, the effect of gravity on the droplet profile cannot be neglected.

Table 1 Experimental conditions

\begin{tabular}{c|c|c}
\hline Evaluation figure & Sliding angle & Evaporation \\
\hline Liquid & \multicolumn{2}{|c}{ Pure Water } \\
\hline Droplet volume & $90 \mu \mathrm{l}$ & $40 \mu \mathrm{l}$ \\
\hline Inclining speed & $1.0 \mathrm{deg} . / \mathrm{s}$ & \\
\hline Environment & Temperature: $20 \mathrm{deg} . \mathrm{C}$, Relative humidity: $50 \%$ \\
\hline
\end{tabular}

\section{Result and discussions}

\subsection{Sliding angle on pillar array}

Figure 9 (a) shows the relationship between $D$ and $\alpha_{A}$ and $\alpha_{R}$. The ratio of the diameter to the pitch, $D / P$, was kept constant. Both $\alpha_{A}$ and $\alpha_{R}$ were almost independent of the change in $D$ in the range of these dimensions. Figure 9 (b) shows the relationship between $P$ and $\alpha_{A}$ 
and $\alpha_{R}$. $D$ was kept constant at $200 \mu \mathrm{m}$. $\alpha_{R}$ decreased with an increase in $P$. Thus, a flat surface has the smallest value of $\alpha_{R}$. On the other hand, $\alpha_{\mathrm{A}}$ was independent of the change in $P$. Thus, a narrow pitch is preferable for evaporation on an inclined surface since the difference between $\alpha_{A}$ and $\alpha_{R}$ increases; thus, pinning distorts the droplet profile.

These results can be explained by pinning. Figure 10 (a) shows the mechanism for the movement of the receding line when the ratio $D / P$ is constant, as shown in Fig. 9 (a). A high density of pillars increases the pinning strength since the length of the contact line surrounding the pillars increases ${ }^{(7)(10)}$. Since the equivalent ratio of the diameter to the pitch implies the equivalent sum of $L$, the forces required to move the receding line $F$ in Eq. (4) are equal, independent of the diameter $\left(F=F^{\prime}\right)$. On the other hand, Fig. 10 (b) shows the movement when the ratio $D / P$ is different as shown in Fig. 9 (b). In this case, the force decreases with an increase in $P$ since the sum of $L$ decreases $\left(F>F^{\prime \prime}\right)$. Thus, it is considered that $\alpha_{R}$ decreased because a low force was required for sliding at a high value of $P$.

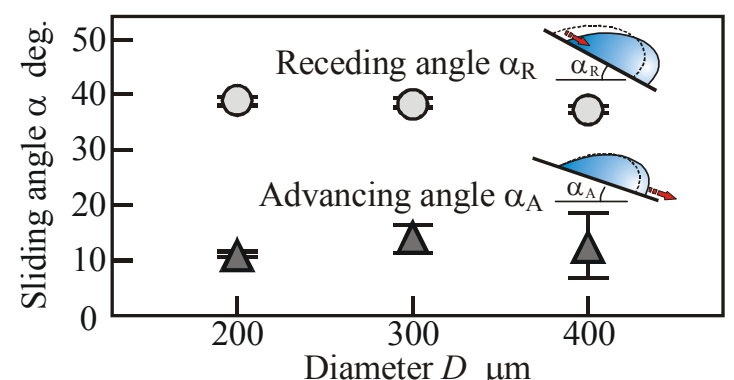

(a) Diameter $(D / P=0.6)$

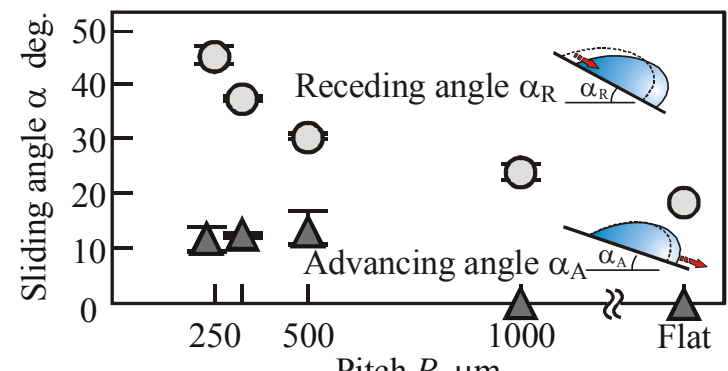

Pitch $P \mu \mathrm{m}$

(b) Pitch $(D=200 \mu \mathrm{m})$

Fig. 9 Sliding angle on pillars $(H=50 \mu \mathrm{m})$

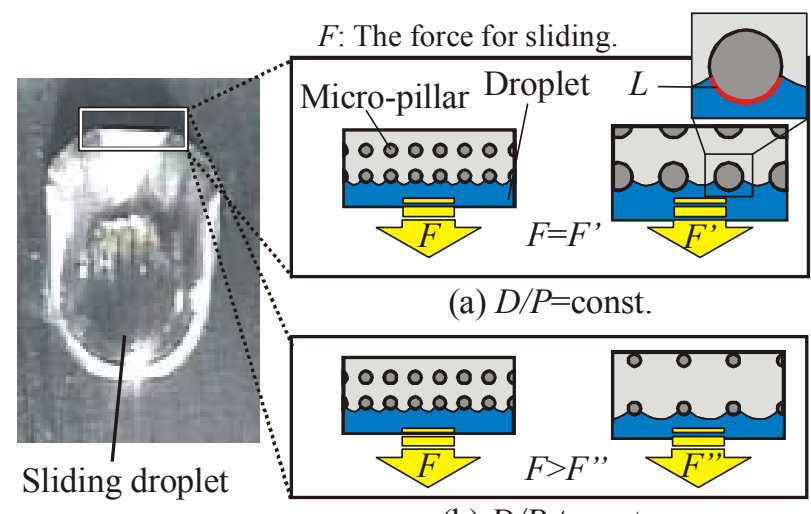

(b) $D / P \neq$ const.

Fig. 10 Pinning at the receding line

\subsection{Evaporation time of droplet on pillar array}

We also investigated the relationship between $P$ and the evaporation time of a droplet, as shown in Fig. 11. The evaporation time decreased with $P$. Thus, it is inferred that the 
evaporation time of a droplet is the longest on a flat surface. Figure 12 shows the top view of a droplet during evaporation. When $P=1000 \mu \mathrm{m}$, the projected area of the droplet began to decrease after $50 \mathrm{~min}$ on the flat surface. However, when $P=340 \mu \mathrm{m}$, the projected area was maintained until the final stage of evaporation. Pinning also causes this behavior. Therefore, the result of Fig. 11 can also be explained by the pinning of the droplet, as in the case of the sliding angle in Fig. 9. As shown in Fig. 10, the force required to move the droplet when $P$ is small is greater than that required when $P$ is large; thus, the projected area should be increased. The evaporation time decreased since the droplet was exposed to air over the large surface area.

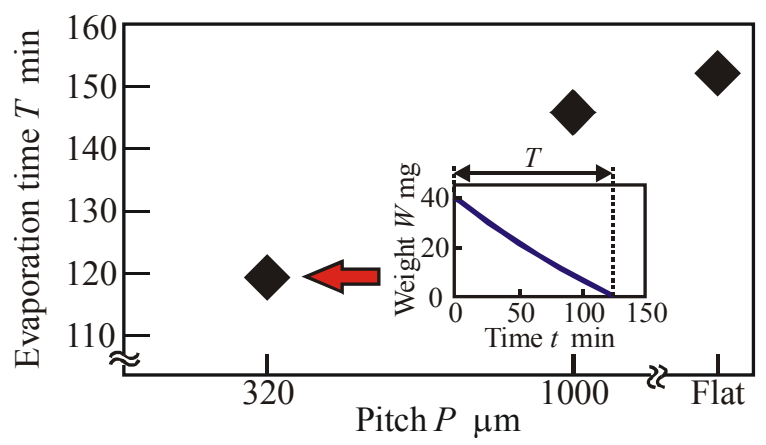

Fig. 11 Relationship between $P$ and evaporation time $(D=200 \mu \mathrm{m}, H=50 \mu \mathrm{m})$

\begin{tabular}{|c|c|c|c|c|c|c|}
\hline$t \min$. & 0 & 50 & 80 & 110 & 130 & 150 \\
\hline Flat & & & & & 6 & \\
\hline$P: 1000 \mu \mathrm{m}$ & & 3 & c. & $\sqrt{3}$ & 6 & \\
\hline$P: 320 \mu \mathrm{m}$ & & c) & & & & \\
\hline
\end{tabular}

Fig. 12 Change in droplet profile with time

\subsection{Design of reticular pattern}

As mentioned above, the sliding angle decreases at a large value of $P$. This indicates that the flat surface has the smallest sliding angle; however, the evaporation time of a droplet on the flat surface is longer than that on a pillared surface. We prepared some reticular patterns, consisting of pillared and flat parts, to achieve a fast evaporation time and a small sliding angle.

Figure 13 shows the specifications of the reticular patterns. $W_{t}$ and $W_{f}$ are the widths of the pillared and flat parts, respectively. The sum of $W_{t}$ and $W_{f}$ was kept constant $\left(W_{t}+W_{f}=\right.$ $1700 \mu \mathrm{m})$. The widths $W_{t}=0 \mu \mathrm{m}$ and $W_{t}=1700 \mu \mathrm{m}$ corresponded to those of the flat surface (no pillars) and fully arrayed surface, respectively. The diameter, pitch, and height of the pillars were also kept constant $(D=100 \mu \mathrm{m}, P=170 \mu \mathrm{m}, H=50 \mu \mathrm{m})$ in order to focus only on the design of the reticular patterns. The patterns were tested using different sliding angles and evaporation times. Figure 14 shows photographs of $40 \mu 1$ droplets on two types of reticular patterns $\left(W_{t}=340 \mu \mathrm{m}\right.$ and $\left.1360 \mu \mathrm{m}\right)$. In these experiments, droplets spread over some pitches of structure, three or four pitches of reticular patterns, thus actual reticular design will be discussed neither on flat surface nor on pillared surface. 


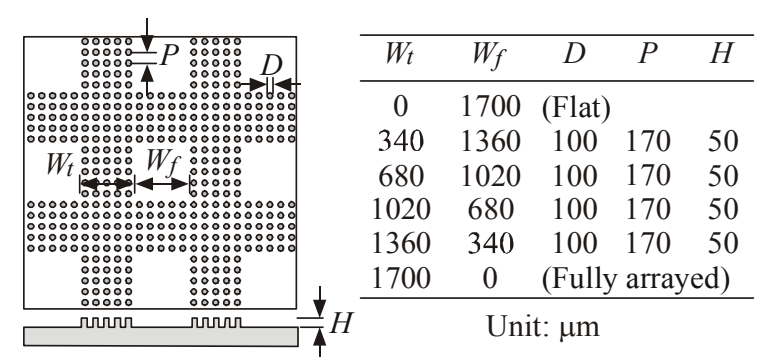

Fig. 13 Schematic illustration and specifications of reticular patterns

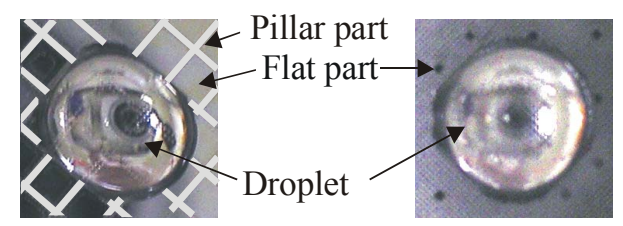

(a) $W_{t}=340 \mu \mathrm{m}$

(b) $W_{f}=1360 \mu \mathrm{m}$

Fig. 14 Droplet on reticular patterns (droplet volume: $40 \mu \mathrm{l}$ )

\subsection{Sliding angle on reticular pattern}

Figure 15 shows the relationship between $W_{t}$ and $\alpha_{A}$ and $\alpha_{R}$. These angles were almost the same as those on the fully arrayed surface $\left(W_{t}=1700 \mu \mathrm{m}\right)$. The flat surface had the smallest sliding angles. Thus, the sliding angles could not be improved by using reticular patterns. This result can be explained on the basis of the fact that the force required to move the receding line is constant when the distance between a pillar and the receding line is constant as shown in Fig. 10.

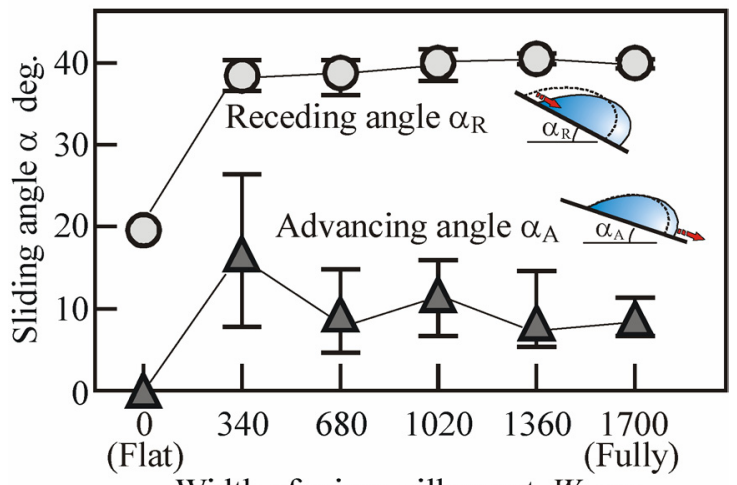

Width of micro-pillar part $W_{t} \mu \mathrm{m}$

Fig. 15 Sliding angles on reticular patterns

Remarkable difference was observed after sliding down of droplet. Figure 16 shows the residual droplet after it slid down the residual patterns $\left(W_{t}=340 \mu \mathrm{m}\right.$ and $\left.1360 \mu \mathrm{m}\right)$. The sliding direction is downward in the figure. The residual water was found only on the pillar part; therefore, the amount of residual water increased with $W_{t}$. This could be explained as follows: the receding line was intermittently pinned when the droplet passed through the pillars as described in Fig. 10, so that the droplet partially remained among them. Thus, a small value of $W_{t}$ is required to remove the droplet efficiently. 


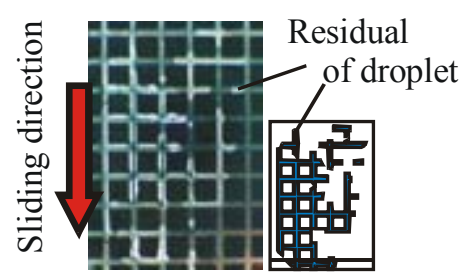

(a) $W_{t}=340 \mu \mathrm{m}$

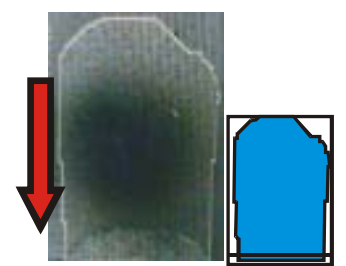

(b) $W_{t}=1700 \mu \mathrm{m}$

Small $W_{t}$ Large

Little Residual of droplet Much

Fig. 16 Relationship between $W_{t}$ and residual droplet after sliding on reticular patterns

\subsection{Evaporation time on reticular pattern}

Figure 17 shows the relationship between $W_{t}$ and the evaporation time. It was found that the evaporation time decreased with $W_{t}$. However, in the case of the flat surface $\left(W_{t}=0\right.$ $\mu \mathrm{m})$, the evaporation time was the longest. Therefore, in this study, an appropriate design of the reticular pattern for reducing the evaporation time is $W_{t}=340 \mu \mathrm{m}$. Figure 18 shows the profile of the droplet during evaporation on the reticular patterns $\left(W_{t}=340 \mu \mathrm{m}\right.$ and 1360 $\mu \mathrm{m})$. The droplet was trapped along the pattern by pinning at $t=120 \mathrm{~min}$ on the surface with $W_{t}=340 \mu \mathrm{m}$. It is thought that the surface area of the droplet at $W_{t}=340 \mu \mathrm{m}$ was larger than that at other values of $W_{t}$, although the projected area was small. It may not be appropriate to use the projected area in the estimation of the surface area, as discussed in section 4.2, since the droplet profile is not hemisphere but complicated spreading over some pitches of reticular patterns.

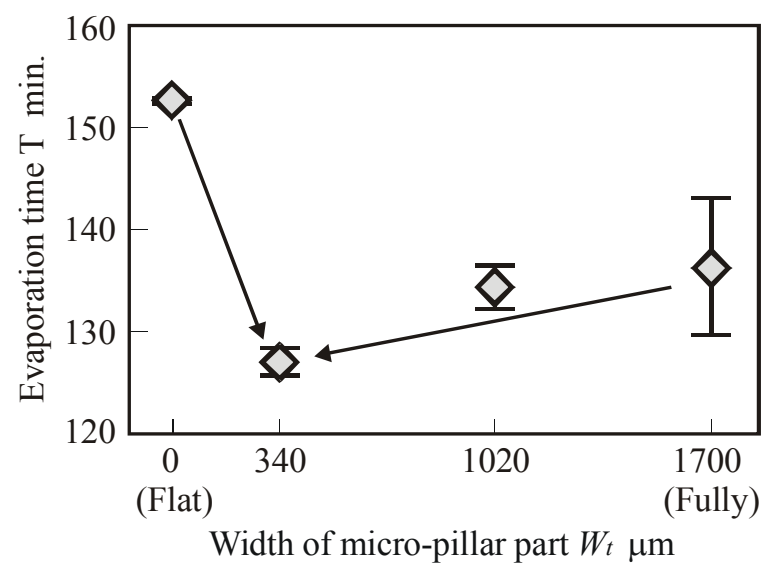

Fig. 17 Relationship between $W_{t}$ and evaporation time

\begin{tabular}{|c|c|c|c|c|c|}
\hline$t \mathrm{~min}$ & 0 & 30 & 60 & 90 & 120 \\
\hline $\begin{array}{c}W_{t}: 340 \mu \mathrm{m} \\
W_{f}: 1360 \mu \mathrm{m}\end{array}$ & & & & & \\
\hline $\begin{array}{r}W_{t}: 1360 \mu \mathrm{m} \\
W_{f}: 340 \mu \mathrm{m}\end{array}$ & & & & 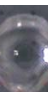 & \\
\hline
\end{tabular}

Fig. 18 Change in droplet profile with time on reticular patterns $\left(W_{t}=340 \mu \mathrm{m}\right.$ and $\left.1360 \mu \mathrm{m}\right)$

\section{Conclusion}

We conducted experiments to design pillared surfaces for quick drying. The results are 
summarized as follows:

(1) The sliding angle decreased with an increase in the pillar pitch, although the evaporation time increased due to the pinning of the droplets.

(2) The evaporation time decreased when the width of the pillar part in the reticular pattern was small, although the sliding angle was large. The small width was effective in the removal of the droplets.

\section{References}

(1) Kim, J., and Kim, C. J., Nanostructured surfaces for dramatic reduction of flow resistance in droplet-based microfluidics, MEMS Conference, IEEE, (2002), pp. 479-482.

(2) Berry, S., Kedzierski, J., and Abedian, B., Low voltage electrowetting using thin fluoroploymer, Journal of Colloid and Interface Science, Vol. 303 (2006), pp. 517-524.

(3) Rico, V., López, C., Borrás, A., Espinos, J. P., and Gónzález-Elipe, A. R., Solar Energy Materials \& Solar Cells, Vol. 90 (2006), pp. 2944-2949.

(4) Kikuchi, A., and Okano, T., Nanostructured designs of biomedical materials: applications of cell sheet engineering to functional regenerative tissues and organs, Journal of Controlled Release, Vol. 101 (2005), pp. 69-84.

(5) Suzuki, S., Nakajima, A., Sakai, M., Song, J.-H., Yoshida, N., Kameshima, Y., and Okada, K., Sliding acceleration of water droplets on a surface coated with fluoroalkylsilane and octadecyltrimethoxysilane, Surface Science, Vol. 600 (2006), pp. 2214-2219.

(6) Ma, M., and Hill, M. R., Superhydrophobic surfaces, Current Opinion in Colloid \& Interface Science, Vol. 11 (2006), pp. 193-202.

(7) de Gennes, P.-G., Brochar-Wyart, F., and Quérér, D., Gouttes, bulles, perles et ondes, Berlin (2003).

(8) Young, T., An essay on the cohesion of fluids, Philosophical Transactions of the Royal Society of London, Vol. 95 (1805), pp. 65-87.

(9) Chan, K. B., and Pierce, S. M., Sessile droplet de-pinning: new life for gravimetric data, Journal of Colloid and Interface Science, Vol. 306 (2007), pp. 187-191.

(10) Nadkarni, G. D., and Garoff, S., An investigation of microscopic aspects of contact angle hysteresis: pinning of the contact line on a sliding defect, Europhysics Letters, Vol. 20 (1992), pp. 523-528.

(11) Jiang, K., Lancaster, M. J., Llamas-Garro, I., and Jin, P., SU-8 Ka-band filter and its microfabrication, Journal of Micromechanics and Microengineering, Vol. 15 (2005), pp. $1522-1526$. 